\title{
GLOBAL PRACTICAL STABILIZATION AND TRACKING FOR AN UNDERACTUATED SHIP - A COMBINED AVERAGING AND BACKSTEPPING APPROACH
}

\author{
K.Y. Pettersen* and H. Nijmeijer** \\ * Department of Engineering Cybernetics \\ Norwegian University of Science and Technology \\ 7034 Trondheim, Norway \\ E-mail: Kristin.Y.Pettersen@itk.ntnu.no \\ ** Faculty of Mathematical Sciences \\ University of Twente \\ P.O. Box 217, 7500 AE Enschede, The Netherlands \\ ** Faculty of Mechanical Engineering \\ Technical University of Eindhoven \\ P.O. Box 513, 5600 MB Eindhoven, The Netherlands \\ E-mail: H.Nijmeijer@math.utwente.nl
}

\begin{abstract}
We solve both the global practical stabilization and tracking problem for an underactuated ship, using a combined integrator backstepping and averaging approach. Exponential convergence to an arbitrarily small neighbourhood of the origin and of the reference trajectory, respectively, is proved. Simulation results are included.

Résumé: Nous résolvons á la fois la stabilisation globale et le problème de poursuite de trajectoire d'un bateau sous-actionné, en utilisant une combinaison entre un "backstepping" et une approche moyenne. La convergence exponentielle vers un voisinage arbitrairement petit de l'origine et de la trajectoire de référence est prouvée. Les résultats de simulation sont inclus.
\end{abstract}

Keywords: Time-varying control, exponential convergence, underactuated surface vessels

\section{INTRODUCTION}

We consider the control of a ship having two independent aft thrusters but no bow or side thrusters. This is a common thruster configuration, for instance many supply vessels have this equipment. We consider two control problems: Position and orientation control is required in many offshore oil field operations, such as drilling, pipe-laying, tanking between ships, diving support, etc. The first control problem considered is therefore to find a feedback control law that asymptotically stabilizes both the position and orientation to desired constant values, using only the two available thrusters. It is not possible to stabilize the ship using a feedback law that is a continuous or discontinuous function of the state only. This follows from results by Brockett (1983), Coron and Rosier (1994) and Zabczyk (1989). The problem is thus not solvable using linearization and linear control theory or classical nonlinear control theory like feedback linearization. In Reyhanoglu (1996) a discontinuous feedback control law is proposed that provides exponential convergence to the desired equilibrium point, under certain assumptions on the initial value. In Pettersen and 
Egeland (1996) a time-varying feedback control law is proposed that provides exponential stability of the desired equilibrium point. However the feedback law only locally stabilizes the desired equilibrium point, and the domain of attraction is not known. In this paper we propose a timevarying feedback control law that provides global exponential practical stability.

The other control problem considered in this paper is the tracking control problem for the underactuated ship. Previous work on this problem has been done by Godhavn (1996), where a control law based on feedback linearization is presented. The control law provides exponential tracking of the position, while the orientation of the ship is not regulated. In this paper we present a feedback control law that tracks both the position and the orientation. The proposed control laws are based on a combined integrator backstepping and averaging approach.

The paper is organized as follows. In Section 2 the model of the ship is presented. In Section 3 a feedback control law is developed that globally practically stabilizes the ship, and simulation results for this stabilizing control law are presented in Section 4. Then a feedback control law for global practical tracking is developed in Section 5 and simulation results are presented in Section 6 .

\section{THE SHIP MODEL}

For the development of the control laws, we use a nonlinear model of the ship based on Fossen (1994). The underactuation leads to a constraint on the acceleration given by the dynamics of the underactuated degree of freedom:

$$
\dot{v}+c u r+d v=0
$$

where $u, v$ and $r$ are the velocities in surge, sway and yaw respectively. The second term represents coriolis and centripetal forces, including added mass effects, while the third term represents the hydrodynamic damping. The dynamics of the underactuated degree of freedom must be considered in the control design, while we assume that the actuated degrees of freedom are controlled by inner control loops such that the corresponding velocities $u$ and $r$ can be considered as control inputs. The kinematics of the ship are described by

$$
\begin{aligned}
\dot{x} & =\cos (\psi) u-\sin (\psi) v \\
\dot{y} & =\sin (\psi) u+\cos (\psi) v \\
\dot{\psi} & =r
\end{aligned}
$$

where $x, y$ and $\psi$ give the position and orientation of the ship in the earth-fixed frame. To obtain simpler, polynomial equations we use the same coordinate transformation as in Pettersen and Egeland (1996)

$$
\begin{aligned}
& z_{1}=\cos (\psi) x+\sin (\psi) y \\
& z_{2}=-\sin (\psi) x+\cos (\psi) y \\
& z_{3}=\psi
\end{aligned}
$$

The resulting model of the ship is then:

$$
\begin{aligned}
\dot{z}_{1} & =u+z_{2} r \\
\dot{z}_{2} & =v-z_{1} r \\
\dot{z}_{3} & =r \\
\dot{v} & =-c u r-d v
\end{aligned}
$$

\section{GLOBAL PRACTICAL STABILIZATION}

For developing a stabilizing feedback control law we use a combined averaging (see for instance Sanders and Verhulst (1985)) and backstepping (see for instance Krstić et al. (1995), Marino and Tomei (1995), Jiang and Nijmeijer (1997)) approach. Define

$$
\begin{aligned}
\alpha^{v}(z) & =-k z_{2} \\
\bar{v} & =v-\alpha^{v}
\end{aligned}
$$

The system equations in the coordinates $\left(z_{1}, z_{2}, z_{3}, \bar{v}\right)$ are then

$$
\begin{aligned}
\dot{z}_{1} & =u+z_{2} r \\
\dot{z}_{2} & =\bar{v}-k z_{2}-z_{1} r \\
\dot{z}_{3} & =r \\
\dot{\bar{v}} & =-c u r-d\left(\bar{v}-k z_{2}\right)+k\left(\bar{v}-k z_{2}-z_{1} r\right)
\end{aligned}
$$

We propose the feedback control law

$$
\begin{aligned}
u= & -k_{1} z_{1}+\gamma\left(\left|z_{1}\right|+\left|z_{2}\right|+\left|z_{3}\right|+|\bar{v}|\right) \sin \left(\frac{t}{\varepsilon}\right) \\
r= & -k_{2} z_{3}+2\left(\frac{k\left(1+\frac{d}{c \gamma}-\frac{k}{c \gamma}\right) z_{2}}{\left(\left|z_{1}\right|+\left|z_{2}\right|+\left|z_{3}\right|+|\bar{v}|\right)}\right. \\
& \left.+\frac{\frac{k_{2}}{\gamma}\left(\frac{k}{c}-k_{1}\right) z_{1} z_{3}+\left(\frac{k}{\gamma c}+k^{v}\right) \bar{v}}{\left(\left|z_{1}\right|+\left|z_{2}\right|+\left|z_{3}\right|+|\bar{v}|\right)}\right) \sin \left(\frac{t}{\varepsilon}\right)
\end{aligned}
$$

where the control parameters $\gamma, k, k_{1}, k_{2}, k^{v}$ are chosen such that for some constant $\beta>0$

$$
\begin{aligned}
k_{1} & >\frac{1}{2} \beta & k & >\frac{1}{2} \beta \\
k_{2} & >\frac{1}{2} \beta & \gamma k^{v} & >\frac{\frac{1}{2} \beta-d}{c}
\end{aligned}
$$

The averaged system equations (Sanders and Verhulst, 1985) of the closed-loop system are then

$$
\begin{aligned}
\dot{z}_{1} & =-k_{1} z_{1}-k_{2} z_{2} z_{3} \\
\dot{z}_{2} & =\bar{v}-k z_{2}+k_{2} z_{1} z_{3} \\
\dot{z}_{3} & =-k_{2} z_{3} \\
\dot{\bar{v}} & =-c k \gamma z_{2}-k^{v} \gamma c \bar{v}-d \bar{v}
\end{aligned}
$$

Consider the Lyapunov function candidate

$$
V(z, \bar{v})=\frac{1}{2} c k \gamma z_{1}^{2}+\frac{1}{2} c k \gamma z_{2}^{2}+\frac{1}{2} z_{3}^{2}+\frac{1}{2} \bar{v}^{2}
$$


The time derivative of $V$ is

$$
\begin{aligned}
\dot{V}(z, \bar{v})= & -c k \gamma k_{1} z_{1}^{2}-c k^{2} \gamma z_{2}^{2} \\
& -k_{2} z_{3}^{2}-\left(c k^{v} \gamma+d\right) \bar{v}^{2}
\end{aligned}
$$

Lyapunov theory then give the following result.

Proposition 1. The origin of the averaged closedloop system (10) is globally exponentially stable.

Definition 1. $\delta(\varepsilon)=O(\varepsilon)$ if there exists positive constants $c_{1}$ and $c_{2}$ such that

$$
|\delta(\varepsilon)| \leq c_{1}|\varepsilon| \quad \forall|\varepsilon|<c_{2}
$$

The time-varying closed-loop system (7-9) satisfies the conditions of Sanders and Verhulst (1985, Theorem 4.2.1), and the following result follows.

Proposition 2. Let $(z, \bar{v})(t)$ be the solution of the closed-loop system $(7-9)$ and $(z, \bar{v})_{\text {av }}(t)$ the solution of the system (10). Then $\forall(z, \bar{v})(0)$

$$
\left\|(z, \bar{v})(t)-(z, \bar{v})_{\mathrm{av}}(t)\right\|=O(\varepsilon) \quad t \in[0, \infty)
$$

The solutions of $(7-9)$ are thus globally uniformly ultimately bounded.

We have thus proved that the closed-loop system converges exponentially to an arbitrarily small ball around the origin.

\section{SIMULATIONS WITH THE STABILIZING CONTROL LAW}

The ship model used in the simulations describes a model ship of scale 1:70, having a mass of $17.6 \mathrm{~kg}$ and a length of $1.2 \mathrm{~m}$. The control inputs to the ship were the force $\tau_{u}=-k_{3}\left(u-u_{c}\right)$ in the surge direction, and the torque $\tau_{r}=-k_{4}\left(r-r_{c}\right)$ in yaw, where $u_{c}$ and $r_{c}$ are the commanded control inputs given in (8-9). The control parameters were chosen as

$$
\begin{array}{rlrl}
k_{1} & =0.5 & k & =0.04 \\
k_{2} & =0.5 & k^{v} & =0.6 \\
k_{3} & =10 & \varepsilon & =1 \\
k_{4} & =10 & \gamma & =1
\end{array}
$$

Initially the ship was at rest, with a deviation of $1 \mathrm{~m}$ in the $y$-direction from the desired position. Figure 1 shows the trajectory of the ship in the $x y$-plane, and Figures 2-3 the time evolution of $\psi$ and $v$ respectively. The simulation shows how the ship converges to a small neighbourhood of the origin.

\section{GLOBAL PRACTICAL TRACKING CONTROL}

In this section we develop a time-varying feedback law that practically stabilizes the system around

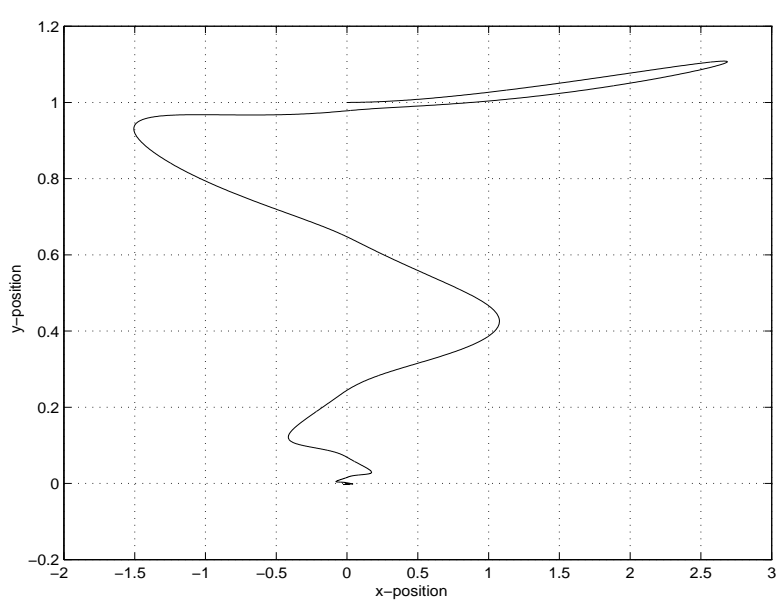

Fig. 1. The trajectory in the $x y$-plane.

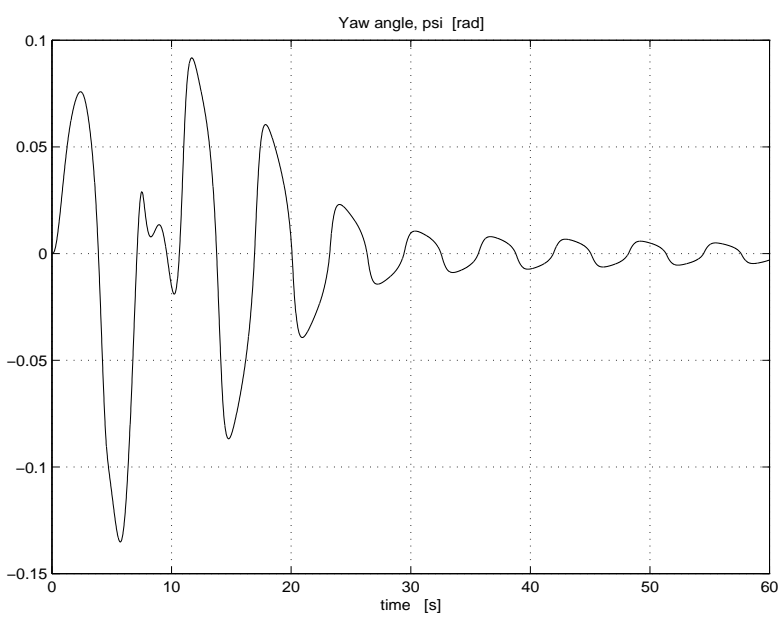

Fig. 2. The time evolution of $\psi[\mathrm{rad}]$.

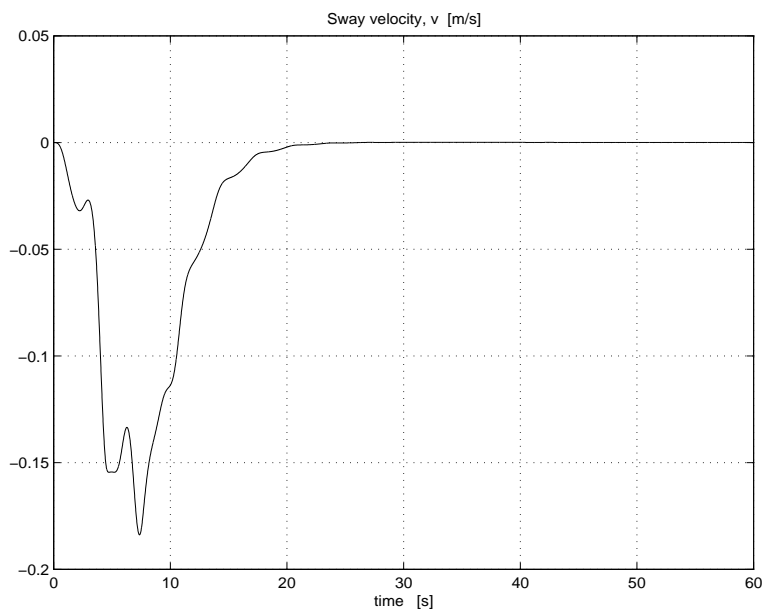

Fig. 3. The time evolution of $v[\mathrm{~m} / \mathrm{s}]$.

the desired trajectory from any initial tracking error. While we consider the tracking problem, the motion planning problem consists of finding paths 
$\left(x_{r}(t), y_{r}(t), \psi_{r}(t)\right)$ which connect a given set of way points. Paths of minimal length, with a given upper bound on curvature and with prescribed initial and terminal positions and tangents are combinations of arcs of circles and line segments. Due to this fact we consider the tracking of trajectories $\left(x_{r}(t), y_{r}(t), \psi_{r}(t)\right)$ for which $u_{r}$ and $r_{r}$ are constants, i.e. the trajectory is a line segment or the arc of a circle. Moreover, no sway velocity is desired, i.e. $v_{r}=0$. The trajectories must thus satisfy the equations

$$
\begin{aligned}
\dot{x}_{r} & =\cos \left(\psi_{r}\right) u_{r} \\
\dot{y}_{r} & =\sin \left(\psi_{r}\right) u_{r} \\
\dot{\psi}_{r} & =r_{r} \\
v_{r} & =0
\end{aligned}
$$

The control problem is thus to find a feedback law that stabilizes the reference trajectory generated by (15).

We define the error coordinates as in Kanayama et al. (1990):

$$
\left[\begin{array}{c}
x_{e} \\
y_{e} \\
\psi_{e} \\
v_{e}
\end{array}\right]=\left[\begin{array}{cccc}
\cos (\psi) & \sin (\psi) & 0 & 0 \\
-\sin (\psi) & \cos (\psi) & 0 & 0 \\
0 & 0 & 1 & 0 \\
0 & 0 & 0 & -1
\end{array}\right]\left[\begin{array}{c}
x_{r}-x \\
y_{r}-y \\
\psi_{r}-\psi \\
-v
\end{array}\right]
$$

The system equations (1-2) are in the error coordinates:

$$
\begin{aligned}
\dot{x}_{e} & =r y_{e}-u+u_{r} \cos \left(\psi_{e}\right) \\
\dot{y}_{e} & =-r x_{e}-v_{e}+u_{r} \sin \left(\psi_{e}\right) \\
\dot{\psi}_{e} & =r_{r}-r \\
\dot{v}_{e} & =-c u r-d v_{e}
\end{aligned}
$$

We then define

$$
\begin{aligned}
\alpha^{v_{e}} & =k y_{e}+u_{r} \sin \left(\psi_{e}\right) \\
\bar{v}_{e} & =v_{e}-\alpha^{v_{e}}
\end{aligned}
$$

The equation for $\dot{\bar{v}}_{e}$ is then

$$
\begin{aligned}
\dot{\bar{v}}_{e}= & -c u r-d\left(\bar{v}_{e}+k y_{e}+u_{r} \sin \left(\psi_{e}\right)\right) \\
& -k\left(-r x_{e}-\bar{v}_{e}-k y_{e}\right)-u_{r} \cos \left(\psi_{e}\right)\left(r_{r}-r\right)
\end{aligned}
$$

We propose the time-varying feedback control law

$$
\begin{aligned}
& u=k_{1} x_{e}+u_{r} \cos \left(\psi_{e}\right) \\
& +\gamma\left(\left|x_{e}\right|+\left|y_{e}\right|+\left|\psi_{e}\right|+\left|\bar{v}_{e}\right|+\left|u_{r} r_{r} \cos \left(\psi_{e}\right)\right|\right) \sin (t / \varepsilon) \\
& r=k_{2} \psi_{e}+r_{r}+2 \sin (t / \varepsilon)( \\
& \quad \frac{k\left(\frac{k}{c \gamma}-\frac{d}{c \gamma}-1\right) y_{e}+\left(k^{v}+\frac{k}{c \gamma}\right) \bar{v}_{e}}{\left(\left|x_{e}\right|+\left|y_{e}\right|+\left|\psi_{e}\right|+\left|\bar{v}_{e}\right|+\left|u_{r} r_{r} \cos \left(\psi_{e}\right)\right|\right)} \\
& +\frac{\frac{k_{2}}{\gamma}\left(\frac{k}{c}-k_{1}\right) x_{e} \psi_{e}+\left(\frac{1}{c}-1\right) \frac{k_{2}}{\gamma} u_{r} \psi_{e} \cos \left(\psi_{e}\right)}{\left(\left|x_{e}\right|+\left|y_{e}\right|+\left|\psi_{e}\right|+\left|\bar{v}_{e}\right|+\left|u_{r} r_{r} \cos \left(\psi_{e}\right)\right|\right)} \\
& \left.+\frac{\left(\frac{k}{c \gamma}-\frac{k_{1}}{\gamma}\right) r_{r} x_{e}-\frac{u_{r} r_{r}}{\gamma} \cos \left(\psi_{e}\right)-\frac{d}{c \gamma} u_{r} \sin \left(\psi_{e}\right)}{\left(\left|x_{e}\right|+\left|y_{e}\right|+\left|\psi_{e}\right|+\left|\bar{v}_{e}\right|+\left|u_{r} r_{r} \cos \left(\psi_{e}\right)\right|\right)}\right)
\end{aligned}
$$

where the control parameters $\gamma, k, k_{1}, k_{2}, k^{v}$ are chosen such that for some constant $\beta>0$

$$
\begin{aligned}
k_{1} & >\frac{1}{2} \beta & k & >\frac{1}{2} \beta \\
k_{2} & >\frac{1}{2} \beta & \gamma k^{v} & >\frac{\frac{1}{2} \beta-d}{c}
\end{aligned}
$$

The averaged system equations of the closed-loop are then

$$
\begin{aligned}
\dot{x}_{e} & =\left(k_{2} \psi_{e}+r_{r}\right) y_{e}-k_{1} x_{e} \\
\dot{y}_{e} & =-\left(k_{2} \psi_{e}+r_{r}\right) x_{e}-\bar{v}_{e}-k y_{e} \\
\dot{\psi}_{e} & =-k_{2} \psi_{e} \\
\dot{v}_{e} & =c k \gamma y_{e}-c k^{v} \gamma \bar{v}_{e}-d \bar{v}_{e}
\end{aligned}
$$

We use the Lyapunov function candidate

$$
\begin{aligned}
& W\left(x_{e}, y_{e}, \psi_{e}, \bar{v}_{e}\right)= \\
& \quad \frac{1}{2} \gamma c k x_{e}^{2}+\frac{1}{2} \gamma c k y_{e}^{2}+\frac{1}{2} \psi_{e}^{2}+\frac{1}{2} \bar{v}_{e}^{2}
\end{aligned}
$$

and find that

$$
\begin{aligned}
\dot{W}\left(x_{e}, y_{e}, \psi_{e}, \bar{v}_{e}\right)= & -\gamma c k k_{1} x_{e}^{2}-\gamma c k^{2} y_{e}^{2} \\
& -k_{2} \psi_{e}^{2}-\left(c k^{v} \gamma+d\right) \bar{v}_{e}^{2}
\end{aligned}
$$

We have thus proved the following result.

Proposition 3. The origin of the averaged closedloop system (23) is globally exponentially stable.

As the time-varying closed-loop system (17)-(22) satisfies the conditions of Sanders and Verhulst (1985, Theorem 4.2.1), we have proved the following result.

Proposition 4. Let $\left(x_{e}, y_{e}, \psi_{e}, \bar{v}_{e}\right)(t)$ be the solution of the system (17)-(22), and let $\left(x_{e}, y_{e}, \psi_{e}\right.$, $\left.\bar{v}_{e}\right)_{\text {av }}(t)$ be the solution of the system (23). Then $\forall\left(x_{e}, y_{e}, \psi_{e}, \bar{v}_{e}\right)(0)$

$$
\begin{gathered}
\left\|\left(x_{e}, y_{e}, \psi_{e}, \bar{v}_{e}\right)(t)-\left(x_{e}, y_{e}, \psi_{e}, \bar{v}_{e}\right)_{\mathrm{av}}(t)\right\| \\
=O(\varepsilon) \quad t \in[0, \infty)
\end{gathered}
$$

Thus the solutions $\left(x_{e}, y_{e}, \psi_{e}, \bar{v}_{e}\right)(t)$ are globally uniformly ultimately bounded.

Remark 1. Note that it is not assumed here that $u_{r} \neq 0$, as has often been assumed in previous work (see e.g. Godhavn (1996), Nakamura and Savant (1992)). In fact, if the desired trajectory is an equilibrium point of the system (1-2), then the tracking controller $(21-22)$ reduces to the stabilizing controller (8-9).

\section{SIMULATIONS, THE TRACKING CONTROL LAW}

The simulations were performed using the same 1:70 ship model as in Section 4. We wanted the ship to move along an arc of a circle with constant speed. The desired yaw rate was $r_{r}=0.01 \mathrm{rad} / \mathrm{s}$ and the desired forward velocity was $u_{r}=0.1 \mathrm{~m} / \mathrm{s}$. The initial state of the reference model (15) was the origin. The desired trajectory, satisfying (15) was then given by

$$
\begin{aligned}
x_{r} & =10 \sin (0.01 t) \\
y_{r} & =10(1-\cos (0.01 t)) \\
\psi_{r} & =0.01 t
\end{aligned}
$$


The control parameters were chosen as

$$
\begin{array}{rlrl}
k_{1} & =0.5 & k & =0.04 \\
k_{2} & =0.5 & k^{v} & =0.6 \\
k_{3} & =10 & \varepsilon & =1 \\
k_{4} & =10 & \gamma & =0.1
\end{array}
$$

The surge and yaw velocities were initially zero. While the initial state of the reference model (15) was the origin, the actual initial state of the ship was

$$
[x, y, \psi, v]=[-1,-1,-0.1,0.01]
$$

Figure 4 shows the trajectory of the ship together with the reference trajectory, in the $x y$-plane. Figure 5 shows the time-evolution of the yaw angle together with the desired yaw angle, and Figure 6 shows the time-evolution of the sway velocity, which has zero as desired value. Then, the value of $\varepsilon$ was changed to $\varepsilon=0.5$. The simulation results are shown in Figures 7-9. We see from the simulations that choosing a smaller value of $\varepsilon$, the system (17) converges to a smaller neighbourhood of the origin. This is in accordance with the result proved in Section 5, that the variables $x_{e}, y_{e}, \psi_{e}$ and $v_{e}$ converge to a ball around the origin that can be made arbitrarily small by choosing $\varepsilon$ sufficiently small.

\section{CONCLUSION}

In this paper the control problems of global practical stabilization and tracking for an underactuated ship have been considered. Two time-varying feedback control laws were derived using a combined integrator backstepping and averaging approach. The trajectories of the controlled ship were proved to converge exponentially to an arbitrarily small neighbourhood of the origin, and of the reference trajectory, respectively. This was illustrated by simulations.

\section{REFERENCES}

Brockett, R.W. (1983). Asymptotic stability and feedback stabilization. In: Differential Geometric Control Theory (R. W. Brockett, R. S. Millman and H. J. Sussmann, Eds.). pp. 181191. Birkhäuser.

Coron, J.-M. and L. Rosier (1994). A relation between continuous time-varying and discontinuous feedback stabilization. J. of Math. Syst. Estimation, and Control 4(1), 67-84.

Fossen, T. I. (1994). Guidance and Control of Ocean Vehicles. John Wiley \& Sons Ltd. Chichester.

Godhavn, J.-M. (1996). Nonlinear tracking of underactuated surface vessels. In: Proc. 35th IEEE Conf. on Decision and Control. Kobe, Japan. pp. 987-991.

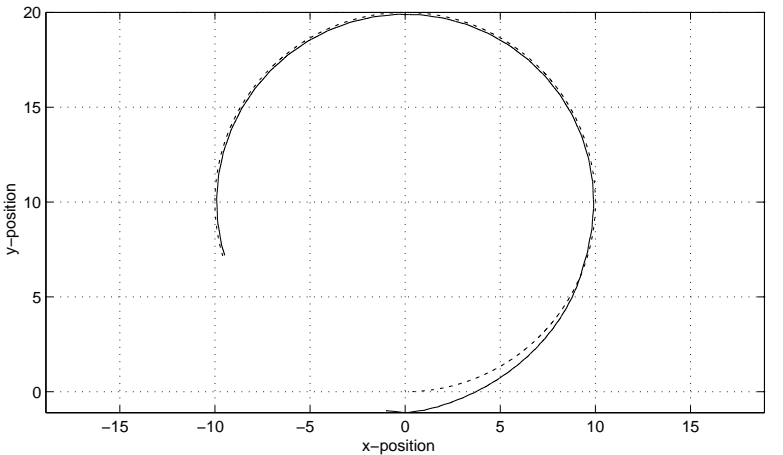

Fig. 4. The actual trajectory (-) and the reference trajectory (- -) in the $x y$-plane, when $\varepsilon=1$.

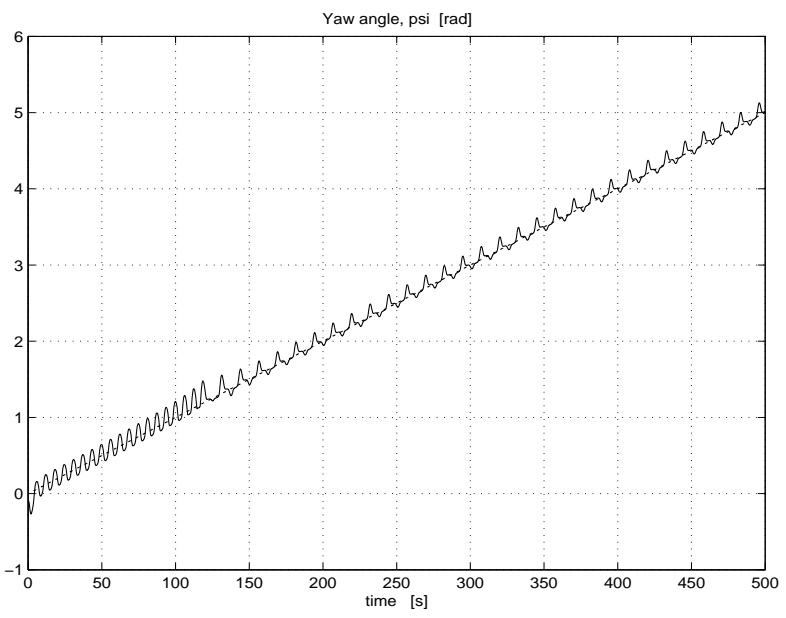

Fig. 5. The time evolution of $\psi(-)$ together with the reference $\psi_{r}(--)$, when $\varepsilon=1$.

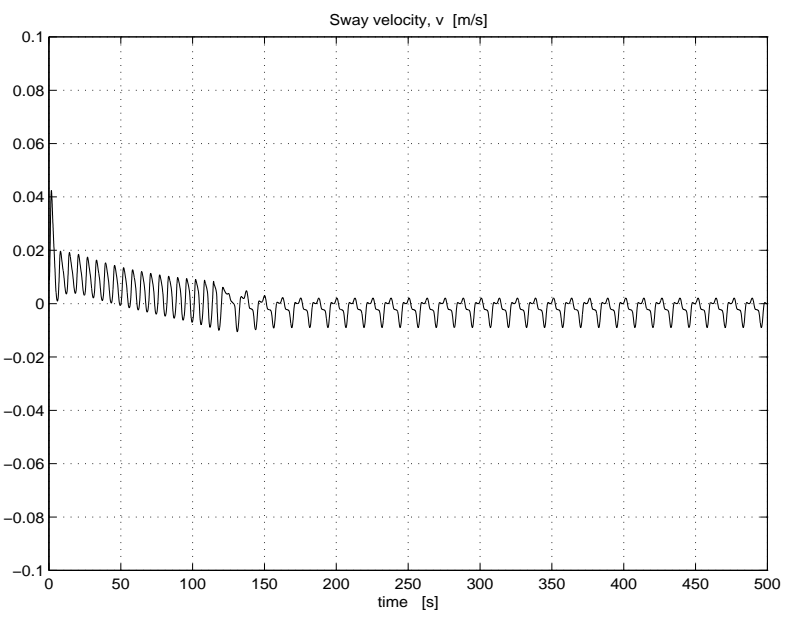

Fig. 6. The time evolution of $v$, when $\varepsilon=1$. 


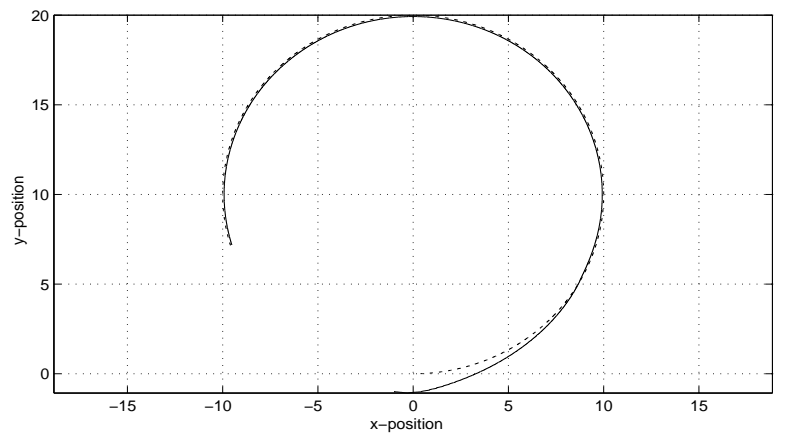

Fig. 7. The actual trajectory $(-)$ and the reference trajectory (- -) in the $x y$-plane, when $\varepsilon=0.5$.

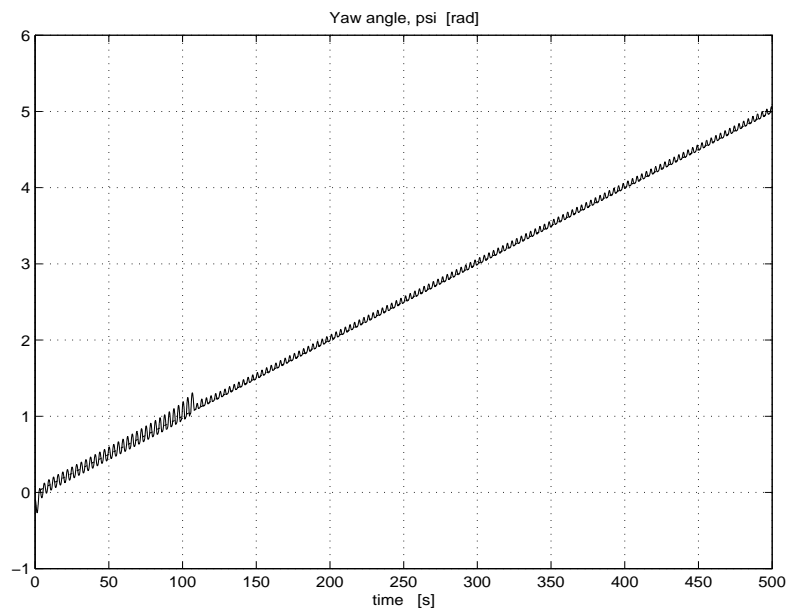

Fig. 8. The time evolution of $\psi(-)$ together with the reference $\psi_{r}(--)$, when $\varepsilon=0.5$.

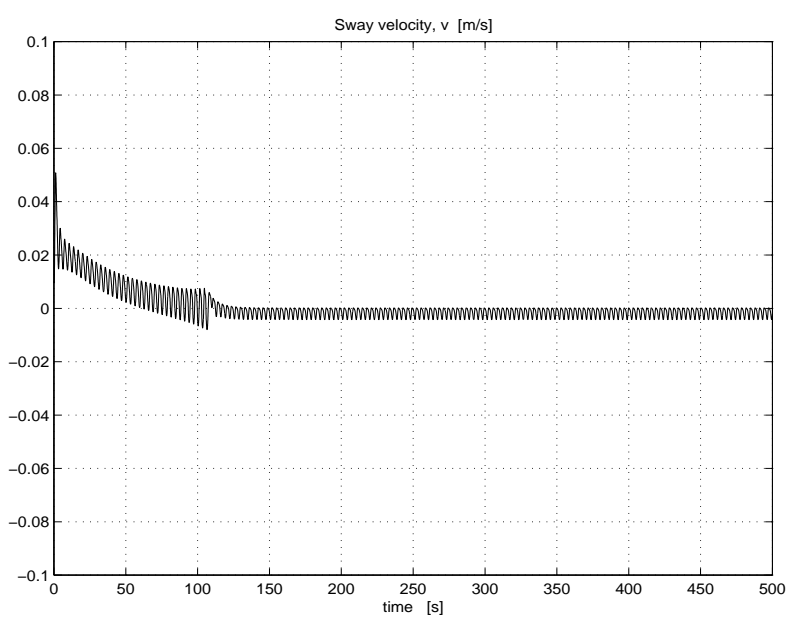

Fig. 9. The time evolution of $v$, when $\varepsilon=0.5$.
Jiang, Z.-P. and H. Nijmeijer (1997). A recursive technique for tracking control of nonholonomic systems in chained form. Technical Report 1397. Univ. of Twente.

Kanayama, Y., Y. Kimura, F. Miyazaki and T. Noguchi (1990). A stable tracking control method for an autonomous mobile robot. In: Proc. 1990 IEEE Int. Conf. on Robotics and Automation. Cincinnati, Ohio. pp. 384-389.

Krstić, M., I. Kanellakopoulos and P. Kokotović (1995). Nonlinear and Adaptive Control Design. Wiley. NY.

Marino, R. and P. Tomei (1995). Nonlinear Control Design. Prentice Hall, Europe.

Nakamura, Y. and S. Savant (1992). Nonlinear tracking control of autonomous underwater vehicle. In: Proc. 1992 IEEE Int. Conf. on Robotics and Automation. Nice, France. pp. A4-A9.

Pettersen, K. Y. and O. Egeland (1996). Exponential stabilization of an underactuated surface vessel. In: Proc. 35th IEEE Conf. on Decision and Control. Kobe, Japan. pp. 967-971.

Reyhanoglu, M. (1996). Control and stabilization of an underactuated surface vessel. In: Proc. 35th IEEE Conf. on Decision and Control. Kobe, Japan. pp. 2371-2376.

Sanders, J.A. and F. Verhulst (1985). Averaging Methods in Nonlinear Dynamical Systems. Springer-Verlag, New York.

Zabczyk, J. (1989). Some comments on stabilizability. Appl. Math. and Optimization 19,19. 